\title{
FUNCTIONS WHICH ARE RESTRICTIONS OF $L^{p}$-MULTIPLIERS
}

BY

\author{
MICHAEL G. COWLING
}

\begin{abstract}
Raouf Doss has given a sufficient condition for a measurable function $\phi$ on a measurable subset $\Lambda$ of an LCA group $\Gamma$ to be the restriction (1.a.e.) to $\Lambda$ of the Fourier transform of a bounded measure, i.e., a Fourier multiplier of type $(1,1)$. We generalise Doss' theorem, and prove that, if the measurable function $\phi$ on $\Lambda$ is approximable on finite subsets of $\Lambda$ by trigonometric polynomials which are Fourier multipliers of type $(p, p)$ on $\Gamma$ of norms no greater than $C$, then $\phi$ is equal locally almost everywhere to the restriction to $\Lambda$ of a Fourier multiplier of type $(p, p)$ and norm no greater than $C$.
\end{abstract}

Before we prove the main theorem, we shall give some definitions and prove a converse of the theorem for continuous multipliers. By $G$ and $\Gamma$, we shall denote dual LCA groups, with Haar measures $d x$ and $d \gamma$ respectively. The group $G$ with the discrete topology is denoted $G_{d}$; its dual is $\bar{\Gamma}$, the Bohr compactification of $\Gamma$. The continuous injection of $\Gamma$ into $\bar{\Gamma}$ is denoted $\beta$; by $\hat{\beta}$ we denote the injection of $G_{d}$ onto $G$. The extended real numbers $p$ and $p^{\prime}$ satisfy $1 \leqslant p \leqslant \infty$ and $p^{-1}+p^{\prime-1}=1 ; L^{p}(G)$ is the usual Lebesgue space on $G . C(G)$ is the space of bounded continuous functions on $G$, with the supremum norm; $C_{0}(G)$ is the space of continuous functions which tend to zero at infinity, and $C_{c}(G)$ is the space of continuous functions with compact supports; both these spaces are normed as subspaces of $C(G)$. The Fourier transform of a function $f$ is denoted $\hat{f} ; A(\Gamma)$ is the space of Fourier transforms of functions in $L^{1}(G)$, with the inherited norm. The subspace of $L^{1} \cap A(G)$ of functions whose Fourier transforms have compact supports is denoted $F A_{c}(G)$.

A (Fourier) multiplier of type $(p, p)$ on $\Gamma$ is a bounded measurable function $\phi$ on $\Gamma$ such that, for any $f, g$ in $C_{c}(G)$,

$$
\left|\int_{\Gamma} \Phi(\gamma) \hat{f}(\gamma) \hat{g}(\gamma) d \gamma\right| \leqslant C\|f\|_{p}\|g\|_{p^{\prime}}
$$

We shall identify such functions which are equal locally almost everywhere (1.a.e.)then the space of (Fourier) multipliers of type $(p, p)$ is a Banach algebra, denoted $M_{p}(\Gamma)$; the norm of $\Phi$ is the least admissible value of $C$ in the inequality (1.1).

Presented to the Society, March 7, 1972; received by the editors July 14, 1973 and, in revised form, January 14, 1975.

AMS(MOS)subject classifications (1970). Primary 43A22.

Key words and phrases. Locally compact abelian group, multipliers, restriction of a multiplier, approximation. 
It is known that

$$
L^{\infty}(\Gamma)=M_{2}(\Gamma) \supseteq M_{p}(\Gamma) \supseteq M_{1}(\Gamma)=B(\Gamma)
$$

$(B(\Gamma)$ is the space of Fourier transforms of bounded measures on $G$, with the inherited norm), and $\|\Phi\|_{\infty} \leqslant\|\Phi\|_{M_{p}} \leqslant\|\Phi\|_{B}$. Consequently, the closure of $A(\Gamma)$ in $M_{p}(\Gamma)$, denoted $m_{p}(\Gamma)$, is a subspace of $C_{0}(\Gamma)$.

The space $M_{p}(\Gamma)$ is the dual space of the space $A_{p}(G)$ introduced by $\mathrm{A}$. Figà-Talamanca [5]. $A_{p}(G)$ is the image under the continuous mapping $f \otimes g \rightarrow$ $f * g$ of the completed projective tensor product $L^{p}(G) \hat{\otimes} L^{p^{\prime}}(G)$ into $C_{0}(G)$. The duality is, when $\Phi \in M_{p}(\Gamma)$ and $h \in A_{p}(G)$,

$$
\langle\Phi, h\rangle=\sum_{1}^{\infty} \int_{\Gamma} \Phi(\gamma) \hat{f}_{n}(\gamma) \hat{g}_{n}(\gamma) d \gamma,
$$

where $f_{n}, g_{n} \in C_{c}(G), \Sigma_{1}^{\infty}\left\|f_{n}\right\|_{p}\left\|g_{n}\right\|_{p^{\prime}}<\infty$, and $h=\Sigma_{1}^{\infty} f_{n} * g_{n}$. It is known that $C_{0}(G)=A_{1}(G) \supseteq A_{p}(G) \supseteq A_{2}(G)=A(G)$, and that the corresponding norm inequalities hold. By $B_{p}(G)$, we denote the algebra of (pointwise) multipliers of $A_{p}(G)$, and give it the obvious norm. If $f \in A_{p}(G),\|f\|_{A_{p}}=\|f\|_{B_{p}}[6]$, so $A_{p}(G)$ is a closed ideal of $B_{p}(G)$.

$$
C(G)=B_{1}(G) \supseteq B_{p}(G) \supseteq B_{2}(G)=B(G),
$$

and again the corresponding norm inequalities hold. If $1<p<\infty$, it is known that $B_{p}(G)$ is the dual space of $m_{p}(\Gamma)$ [7]. The duality is, for $h \in B_{p}(G)$ and $\Phi \in m_{p}(\Gamma)$,

$$
\langle\Phi, h\rangle=\lim _{n \rightarrow \infty} \hat{\Phi}_{n} * h_{v}(0),
$$

where $\Phi_{n} \in A(\Gamma)$ and $\left\|\Phi_{n}-\Phi\right\|_{M_{p}} \rightarrow 0$ as $n \rightarrow \infty$, and $h_{v}$ is the reflection of $h$ : $h_{v}(x)=h(-x)$. This duality coincides with that of (1.2) when $\Phi \in m_{p}(\Gamma)$ and $h \in A_{p}(G)$. We note that one can (and we shall) define $\langle\Phi, h\rangle$ meaningfully for larger sets than we have done.

Our first theorem concerns the local behaviour of multipliers, generalizing Theorem 1 of [2]. Let $K$ be a compact subset of $\Gamma$, and let $m_{p}(K)$ be the algebra of restrictions to $K$ of elements of $m_{p}(\Gamma)$, defined to be the quotient $m_{p}(\Gamma)$ modulo the ideal $i(K)$ of $m_{p}(\Gamma)$-functions which vanish on $K$. Consider the mapping $\beta^{*}: m_{p}(\bar{\Gamma}) \rightarrow M_{p} \cap C(\Gamma)$ defined by the rule $\beta^{*}$ : $\phi \rightarrow \phi \circ \beta$; Lohoué [8] and Saeki [9] have shown that $\beta^{*}$ is norm-decreasing. Further, if $\phi \in m_{p}(\bar{\Gamma})$, there exist $\phi_{n}$ in $A(\bar{\Gamma})$ such that $\left\|\phi_{n}-\phi\right\|_{M_{p}(\bar{\Gamma})} \rightarrow 0$, so $\psi \cdot \beta^{*} \phi_{n} \in A(\Gamma)$ and $\left\|\psi \cdot \beta^{*} \phi_{n}-\psi \cdot \beta^{*} \phi\right\|_{M_{p}(\Gamma)} \rightarrow 0$, where $\psi \in A(\Gamma)$ and $\psi$ takes the value one on $K$, i.e., for any $\phi$ in $m_{p}(\Gamma)$ there exists $\phi^{\prime}$ in $m_{p}(\Gamma)$ such that $\beta^{*} \phi$ and $\phi^{\prime}$ are equal on $K$. Finally, if $\phi \in i(\beta(K))$, then $\beta^{*} \phi$ vanishes on $K$. Therefore $\beta^{*}$ induces a quotient map denoted $\beta_{q}^{*}$, from $m_{p}(\beta(K))$ into $m_{p}(K)$. It is easily seen that $\beta_{q}^{*}$ is one-to-one and decreases norms.

Let $B_{p}(K)$ be the annihilator of $i(K)$ in $B_{p}(G)$. Consider the mapping $\hat{\beta}^{*}$ : 
$B_{p}(G) \rightarrow B_{p}\left(G_{d}\right)$, defined by the rule $\hat{\beta}^{*}: f \rightarrow f \circ \hat{\beta}$, and its restriction to $B_{p}(K)$. Lohoué [8] has shown that $\hat{\beta}^{*}$ is an isometry; further, it is readily checked that, if $f \in B_{p}(G)$ and $\phi \in m_{p}(\bar{\Gamma}),\left\langle\phi, \hat{\beta}^{*} f\right\rangle=\left\langle\beta^{*} \phi, f\right\rangle$ (where we interpret $\beta^{*} \phi$ as $\lim _{n \rightarrow \infty} \beta^{*} \phi_{n}$, where $\phi_{n} \in A(\Gamma)$ and $\left.\lim _{n \rightarrow \infty}\left\|\phi-\phi_{n}\right\|_{M_{p}}=0\right)$. Therefore the restriction of $\hat{\beta}^{*}$ to $B_{p}(K)$ maps into $B_{p}(\beta(K))$, and is the adjoint map of the quotient map $\beta_{q}^{*}$ (in the cases where $q=1$ or $\infty$, the restriction map extends uniquely to the adjoint map of $\beta_{q}^{*}$ and this adjoint map is an isometry). A monomorphism whose adjoint is an isometry is itself an isometry [4, VI. 6]; we have therefore proved:

THEOREM 1. The algebra of restrictions of $m_{p}(\Gamma)$-multipliers to the compact subset $K$ of $\Gamma$ is isomorphic and isometric to the algebra of restrictions of $m_{p}(\bar{\Gamma})$ multipliers to $\beta(K)$.

COROllaRY. An $m_{p}(\Gamma)$-multiplier $\phi$ may be approximated on finite subsets of $\Gamma$ by trigonometric polynomials whose norms, as multipliers of type $(p, p)$ on $\Gamma$, are bounded by the $(p, p)$-multiplier norm of $\phi$.

This follows from a theorem of Lohoue [8] and Saeki [9], which states that, for $\Phi \in M_{p} \cap C(\bar{\Gamma}),\|\Phi \circ \beta\|_{M_{p}}=\|\Phi\|_{M_{p}}$. We may extend this corollary to any continuous multiplier $\phi$ using several results of Lohoue [8]. Let $\Lambda$ be a finite subset of $\Gamma$; denote by $\delta$ the continuous map of $\Gamma_{d}$ onto $\Gamma$ and by $\hat{\delta}$ the injection of $G$ into $\bar{G}$, then $\phi \circ \delta \in M_{p}\left(\Gamma_{d}\right)$, and further, there exists a net of measures $\left(\mu_{\alpha}\right)_{\alpha \in A}$ supported in finite subsets of $\hat{\delta}(G)$ such that, for every $f \in A_{p}(\bar{G})$, $\lim _{A}\left\langle\hat{\mu}_{\alpha}, f\right\rangle=\langle\phi \circ \delta, f\rangle$ and $\left\|\hat{\mu}_{\alpha}\right\|_{M_{p}} \leqslant\|\phi \circ \delta\|_{M_{p}}$. If we consider $f \stackrel{\in}{\in} \delta^{-1}(\Lambda)$, we see that $\lim _{A}\left\langle\hat{\mu}_{\alpha}, \delta^{-1}(\gamma)\right\rangle=\phi(\gamma)$ for $\gamma \in \Lambda$, so defining $\phi_{\alpha}$ by the rule $\phi_{\alpha}$ 。 $\delta=\hat{\mu}_{\alpha}$, we have a net $\left(\phi_{\alpha}\right)_{\alpha \in A}$ of trigonometric polynomials on $\Gamma$ such that $\lim _{A} \phi_{\alpha}(\gamma)=\phi(\gamma)$ for all $\gamma \in \Lambda$ and $\left\|\phi_{\alpha}\right\|_{M_{p}} \leqslant\|\phi\|_{M_{p}}$; the corollary therefore holds for continuous multipliers.

The author [1] has shown also that the algebra of restrictions of $M_{p} \cap$ $C(\Gamma)$-functions to the compact subset $K$ of $\Gamma$ is isomorphic and isometric to the algebra of restrictions of $M_{p} \cap C(\bar{\Gamma})$-functions to $\beta(K)$. To reproduce the proof would obscure the essence of this paper, namely, Theorem 2 , which follows directly. Theorem 2 is a generalisation of the main theorem of Doss' paper [3], to which this paper owes much.

THEOREM 2. Let $\phi$ be a measurable function on the measurable subset $\Lambda$ of $\Gamma$. If $\phi$ can be uniformly approximated on finite subsets of $\Lambda$ by trigonometric polynomials $t$ such that $\|t\|_{M_{p}} \leqslant C$, then $\phi$ is the restriction tc $\Lambda$ of a function $\Phi$ in $M_{p}(\Gamma)$ such that $\|\Phi\|_{M_{p}} \leqslant C$.

Proof. The theorem is trivial if $\Lambda$ is locally null, so we shall assume the contrary; further, we observe that the hypotheses of the theorem imply that $\phi$ is 
bounded (by $C$ ). Because $M_{p}(\Gamma)$ is the dual space of $A_{p}(G)$, it suffices to show that, if $f$ is in $L^{\infty}(\Gamma)$ and vanishes off the compact subset $\Lambda_{0}$ of $\Lambda$,

$$
\left|\int_{\Lambda} d \gamma \phi(\gamma) f(\gamma)\right| \leqslant C\|\hat{f}\|_{A_{p}}(G) \text {. }
$$

For the Hahn-Banach theorem then shows that there is a multiplier $\Phi$ in $M_{p}(\Gamma)$ such that $\|\Phi\|_{M_{p}} \leqslant C$ and

$$
\int_{\Lambda} d \gamma \Phi(\gamma) f(\gamma)=\int_{\Lambda} d \gamma \phi(\gamma) f(\gamma)
$$

for any $f$ in $L^{\infty}(\Gamma)$ vanishing off some compact subset of $\Lambda$. It follows that $\phi=$ $\Phi$ (locally almost everywhere) on $\Lambda$. For convenience, we shall extend $\phi$ to a function $\phi$ on $\Gamma$ by taking $\phi$ to be zero off $\Lambda$.

A key observation to be made is that, if $f$ is in $L^{\infty}(\Gamma)$ and vanishes off the compact subset $\Lambda_{0}$ of $\Lambda$, then

$$
\int_{\Lambda_{0}} d \gamma \phi(\gamma) f(\gamma)=(\phi f)^{\wedge}(0) \doteqdot \int d x(\phi f)^{\wedge}(x) u(x),
$$

where $u$ is a nonnegative $A_{c}(G)$-function with support $V$ "close" to 0 in $G$, and $u$ also satisfies the condition that $\int_{V} d x u(x)=1$. In fact, if $V$ is "small enough",

$$
\int_{\Lambda_{0}} d \gamma \phi(\gamma) f(\gamma) \doteqdot \int d x(\phi f)^{\wedge}(x) \delta(x) u(x)
$$

for any $\delta$ in $\Lambda_{0}$, since, as $x$ tends to 0 in $G, \delta(x)$ tends to 1 uniformly for $\delta$ in the compact set $\Lambda_{0}$. This approximation is needed for technical reasons which will become apparent later.

By Theorem 1 (the case where $p=1$ and $\beta$ is the injection of $G$ into its Bohr compactification $\bar{G}$ ), $u$ may be written as an absolutely convergent trigonometric series $\Sigma_{1}^{\infty} b_{n} \delta_{n}$ on an arbitrarily large compact set $K_{0}$. We have then that

$$
\begin{aligned}
\int_{\Lambda_{0}} d \gamma \phi(\gamma) f(\gamma) & \doteqdot \int_{K_{0}} d x(\phi f)^{\wedge}(x) \delta(x) \sum_{1}^{\infty} b_{n} \delta_{n}(x) \\
& =\int_{K_{0}} d x(\phi f)^{\wedge}(x) \sum_{1}^{\infty} b_{n}\left(\delta+\delta_{n}\right)(x)
\end{aligned}
$$

$\left(\delta+\delta_{n}\right)$ being the group theoretic sum of $\delta$ and $\delta_{n}$.

If it happened that $(\phi f)^{\wedge}$ vanished off $K_{0}$, we could write, using the inversion theorem,

$$
\int_{\Lambda_{0}} d \gamma \phi(\gamma) f(\gamma) \doteqdot \sum_{1}^{\infty} b_{n} \phi\left(\delta+\delta_{n}\right) f\left(\delta+\delta_{n}\right) \doteqdot \sum_{1}^{N} b_{n} \phi\left(\delta+\delta_{n}\right) f\left(\delta+\delta_{n}\right)
$$

for large $N$, because $\Sigma_{1}^{\infty}\left|b_{n}\right|<\infty$. In the formal proof below, the function $(\phi \cdot f)$ is regularised to $(\phi f) * k$, where $\hat{k}$ is supported in $K_{0}$, in order to validate the use of the inversion theorem.

Since $\phi$ can be approximated on the finite subset $\left\{\delta+\delta_{1}, \ldots, \delta+\delta_{N}\right\} \cap$ $\Lambda$ of $\Lambda$ by trigonometric polynomials $t$ with $\|t\|_{M_{p}}$ no greater than $C$, 


$$
\begin{aligned}
\left|\int_{\Lambda_{0}} d \gamma \phi(\gamma) f(\gamma)\right| & \doteqdot\left|\sum_{1}^{N} b_{n} \phi\left(\delta+\delta_{n}\right) f\left(\delta+\delta_{n}\right)\right| \\
& \doteqdot\left|\sum_{1}^{N} b_{n} t\left(\delta+\delta_{n}\right) f\left(\delta+\delta_{n}\right)\right| \\
& \leqslant C\left\|\mid \sum_{1}^{N} b_{n} f\left(\delta+\delta_{n}\right)\left(\delta+\delta_{n}\right)\right\|_{B_{p}(G)}
\end{aligned}
$$

The essence of the formal proof is the estimation of

$$
\left\|\sum_{1}^{N} b_{n} f\left(\delta+\delta_{n}\right)\left(\delta+\delta_{n}\right)\right\|_{B_{p}} .
$$

All we really need to show is that, for any small positive $\epsilon$,

$$
\left\|\sum_{1}^{N} b_{n} f\left(\delta+\delta_{n}\right)\left(\delta+\delta_{n}\right)\right\|_{B_{p}(G)} \leqslant\|\hat{f}\|_{A_{p}(G)}+\epsilon,
$$

for some $\delta$ in $\Lambda_{0}$ because then (2.1) can be established. The proof of this inequality involves regularisation of $f$ to $f * k$, where $k$ is in $F A_{c}(\Gamma)$.

The regularisation of $\phi f$ and $f$ necessitates that we obtain integral estimates for $(\phi f)^{\prime}(0)$ and $\left\|\Sigma_{1}^{N} b_{n} f\left(\delta+\delta_{n}\right)\left(\delta+\delta_{n}\right)\right\|_{B_{p}}$. Rather than prove (2.2) and (2.3) directly, we are obliged to first establish that

$$
\int_{\Lambda_{0}} d \delta\left|\int_{\Lambda_{0}}^{\cdot} d \gamma \phi(\gamma) f(\gamma)-\sum_{1}^{N} b_{n} \phi\left(\delta+\delta_{n}\right) f\left(\delta+\delta_{n}\right)\right|<\epsilon
$$

and

$$
\int_{\Lambda_{0}} d \delta\left\|\sum_{1}^{N} b_{n} f\left(\delta+\delta_{n}\right)\left(\delta+\delta_{n}\right)\right\|_{B_{p}(G)} \leqslant \mu\left(\Lambda_{0}\right)\|\hat{f}\|_{A_{p}(G)}+\epsilon,
$$

where $\mu\left(\Lambda_{0}\right)$ is the Haar measure of $\Lambda_{0}$. From these "randomised" estimates, we deduce the "pointwise" estimates (2.2) and (2.3) by measure theoretic arguments.

Lemma 1 shows how to construct a particular trigonometric series $\Sigma b_{n} \delta_{n}$. Lemma 2 establishes the integral estimates indicated above. This proof relies on the fact that the polynomial $\Sigma b_{n} \delta_{n}$ of Lemma 1 can be chosen with sufficiently weak dependence on $\phi$ and $f$ to permit regularisation of $f$ and $\phi f$.

LEMmA 1. Let $G_{1}$ be an LCA group of the form $R^{a} \oplus T^{b} \oplus D$, where a and $b$ are nonnegative integers and $D$ is a discrete group. Let $V_{0}$ be a neighborhood of 0 in $G_{1}$ which is a direct sum of compact symmetric neighborhoods of 0 in the $R$ and $T$ summands occurring in $G_{1}$, and of the neighbourhood $\{0\}$ of 0 in $D$. Denote by $u$ the function $m\left(V_{0}\right)^{-2} \chi_{V_{0}} * \chi_{V_{0}}$ on $G_{1}$, where $m$ and * 
represent the Haar measure and convolution on $G_{1}$ respectively. Then

(a) $u \geqslant 0$,

(b) $u$ vanishes outside $V_{0}+V_{0}$,

(c) $\int_{G_{1}} d x u(x)=1$.

To any compact set $K_{0}$ containing $V_{0}+V_{0}$, we can associate a trigonometric series $q$ on $G_{1}: q=\Sigma_{1}^{\infty} b_{n} \gamma_{n}$ ( $\gamma_{n}$ in the dual group $\Gamma_{1}$ of $\left.G_{1}\right)$ such that

(d) $\Sigma_{1}^{\infty}\left|b_{n}\right|=m\left(V_{0}\right)^{-1}$,

(e) $u(x)=\Sigma_{1}^{\infty} b_{n} \gamma_{n}(x) \quad \forall x \in K_{0}$.

Further, denoting by $\bar{m}$ and $\bar{*}$ the Haar measure and convolution on the Bohr compactification $\bar{G}_{1}$ of $G_{1}$, we can write the unique continuous extension $\bar{q}$ of the almost periodic function $q$ on $G_{1}$ to $\bar{G}_{1}$ in the following manner:

(f) $\bar{q}=m\left(V_{0}\right)^{-1} \bar{m}(U)^{-1} \chi_{U}{ }^{*} \chi_{U}$, where $U$ is a closed symmetric subset of $\bar{G}_{1}$ such that

(g) $\int_{K_{0}} d x \chi_{U}(\bar{y}+x) \leqslant m\left(V_{0}\right) \forall \bar{y} \in \bar{G}_{1}$; here we regard $G_{1}$ as a dense subgroup of $\bar{G}_{1}$.

Proof. Case (i). $G_{1}=T$. The group $T$ is its own Bohr compactification. Let $U$ be the set $V_{0}$ and let $\Sigma_{1}^{\infty} b_{n} \gamma_{n}$ be the Fourier series of $u$. Evidently $u$, $\Sigma_{1}^{\infty} b_{n} \gamma_{n}$ and $U$ satisfy the conditions of the lemma.

Case (ii). $G_{1}=R$. Take an interval $[-I / 2, I / 2)$ containing $K_{0}$ in its interior, and identify this interval with the quotient group $R / I Z$. The injection $\beta$ of $R$ into $\bar{R}$ induces a homeomorphic isomorphism $\beta_{0}$ of $R / I Z$ onto the quotient group $\bar{R} / H$, where $H$ is the closure in $\bar{R}$ of $\beta(I Z)$. We denote by $\pi_{0}$ and $\pi$ the projections of $R$ onto $R / I Z$ and $\bar{R}$ onto $\bar{R} / H$ respectively; then the following diagram commutes:

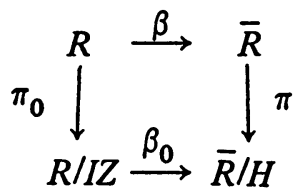

Define $U$ to be the subset $\pi^{-1} \beta_{0} \pi_{0}\left(V_{0}\right)$, and let $q$ be the trigonometric series $\Sigma_{1}^{\infty} b_{n} \gamma_{n}$ on $R$ such that $\bar{q}=m\left(V_{0}\right)^{-1} \bar{m}(U)^{-1} \chi_{U} \bar{*} \chi_{U}$.

We observe that an equivalent expression for $U$ is $\chi_{U}=\Sigma_{x \in I Z} T_{x} \chi_{V_{0}}$, whence, for any $y$ in $R$

$$
\int_{K_{0}} d x \chi_{U}(x+y) \leqslant m\left(V_{0}\right) .
$$

Proof of the inequality $(\mathrm{g})$ of the lemma is similar.

The series $q$ is independent of the particular choice of Haar measure on $\bar{R}$. For the moment, we shall assume that the Haar measure of $[-I / 2, I / 2)$ is equal to the total mass of $R / I Z$, that $\beta_{0}$ is measure preserving, and that the Haar measures of $\bar{R}$ and $\bar{R} / H$ are also equal. Then $m\left(V_{0}\right)=\bar{m}(U)$, and for any $x$ in $R$, 


$$
\begin{aligned}
q(x) & =\bar{q}(\beta(x))=m\left(V_{0}\right)^{-2} \chi_{U} \bar{*} \chi_{U}(\beta(x)) \\
& =m\left(V_{0}\right)^{-2}\left[\chi_{\beta_{0} \pi_{0}\left(V_{0}\right)} * \chi_{\beta_{0} \pi_{0}\left(V_{0}\right)}\right] \circ \pi(\beta(x)) \\
& =m\left(V_{0}\right)^{-2}\left[\chi_{\beta_{0} \pi_{0}\left(V_{0}\right)} * \chi_{\beta_{0} \pi_{0}}\left(V_{0}\right)\right]\left(\beta_{0} \pi_{0}(x)\right) \\
& =m\left(V_{0}\right)^{-2}\left[\chi_{\pi_{0}\left(V_{0}\right)} * \chi_{\pi_{0}\left(V_{0}\right)}\right]\left(\pi_{0}(x)\right) .
\end{aligned}
$$

We conclude that $q$ is periodic, with period $I$, and that

$$
q(x)=m\left(V_{0}\right)^{-2} \chi_{V_{0}} * \chi_{V_{0}}(x), \quad x \in[-I / 2, I / 2) .
$$

The function $q$ and set $U$ therefore satisfy the conditions of the lemma.

Case (iii). $G_{1}=D$. Choose a neighbourhood $W$ of 0 in $\bar{D}$ such that $W$ meets the finite set $\beta\left(K_{0}-K_{0}\right)$ in just the point 0 . Let $\dot{U}$ be a compact symmetric neighbourhood of 0 in $\bar{D}$ such that $U-U \subseteq W$. Take $\bar{q}$ to be the function on $D$ given. by the formula:

$$
\bar{q}=m\left(V_{0}\right)^{-1} \bar{m}(U)^{-1} \chi_{U} \bar{*} \chi_{U} .
$$

By the construction of $U,(\mathrm{~g})$ is satisfied, for if $\bar{y}_{1}$ and $\bar{y}_{2}$ both lie in $\beta\left(K_{0}\right) \cap$ $(\bar{x}+U)$ for some $\bar{x}$ in $\bar{D}$, then

$$
\bar{y}_{1}-\bar{y}_{2} \in \beta\left(K_{0}-K_{0}\right) \cap(U-U)=\{0\} .
$$

Since $V_{0}$ is just the point $\{0\}$ of $D$,

$$
\int_{K_{0}} d x \chi_{U}(x+\bar{y})=m\left(K_{0} \cap \beta^{-1}(U-\bar{y})\right) \leqslant m(\{0\})=m\left(V_{0}\right) .
$$

Moreover, $\operatorname{supp}(q) \subseteq W$ since $U$ is symmetric, and so $q(x)=0$ for all $x$ in $K \backslash\{0\}$. Further, $q(0)=m\left(V_{0}\right)^{-1}$. Now $u$ is just the function $m\left(V_{0}\right)^{-2} \chi_{V_{0}} *$ $\chi_{V_{0}}$ on $D$, i.e. $u=m\left(V_{0}\right)^{-1} \chi_{V_{0}}$. The conditions of the lemma are therefore satisfied.

Case (iv). $G_{1}=R^{a} \oplus T^{b} \oplus D$. We view $\bar{G}_{1}$ as the direct sum $\bar{R}^{a} \oplus T^{b} \oplus$ $\bar{D}$. Any compact neighbourhood $K_{0}$ of 0 in $G_{1}$ can be contained in another of the form

$$
[(1-I) / 2,(I-1) / 2)^{a} \oplus T^{b} \oplus K_{1}
$$

in $G_{1}$. Construct $U, q$ and $u$ for each of the summands, using this latter neighbourhood, and identifying $[-I / 2, I / 2)$ with $R / I Z$ in each of the $R$-summands. Take $u$ and $q$ on $G_{1}$ and $U$ in $\bar{G}_{1}$ to be the products of those $u, q$ and $U$ constructed in the summands.

Our next lemma is the core of the theorem. As we have already explained, the result claimed can be deduced quite easily from the integral estimates obtained. 
LEMMA 2. Let $\Gamma$ be an LCA group with dual $G$. Let $\phi$ and $f$ be bounded measurable functions on $\Gamma$, with $f$ vanishing off the compact subset $\Lambda_{0}$ of $\Gamma$. Suppose $\epsilon$ is a given, arbitrarily small, positive quantity. Then, for a certain polynomial $\Sigma_{1}^{N} b_{n} \gamma_{n}$, the following estimates hold:

$$
\int_{\Lambda_{0}} d \gamma\left|\sum_{1}^{N} b_{n} \phi\left(\gamma+\gamma_{n}\right) f\left(\gamma+\gamma_{n}\right)-(\phi f)^{\wedge}(0)\right|<\epsilon
$$

and

$$
\int_{\Lambda_{n}} d \gamma\left\|\sum_{1}^{N} b_{n} f\left(\gamma+\gamma_{n}\right)\left(\gamma+\gamma_{n}\right)\right\|_{B_{p}(G)} \leqslant \mu\left(\Lambda_{0}\right)\|\hat{f}\|_{A_{p}(G)}+\epsilon,
$$

where $\mu$ is the Haar measure on $\Gamma$.

Proof. Extend $\Lambda_{0}$ to a compact neighbourhood $\Lambda_{1}$ of 0 in $\Gamma$, and let $\Gamma_{1}$ be the open subgroup of $\Gamma$ generated by $\Lambda_{1}$. We take the Haar measure on $\Gamma_{1}$ to be the restriction to $\Gamma_{1}$ of that on $\Gamma$. Let $H_{1}$ be the annihilator of $\Gamma_{1}$ in $G$, and take $G_{1}$ to be the quotient group $G / H_{1}$. From the structure theorem for compactly generated LCA groups, $G_{1}$, which is the dual of $\Gamma_{1}$, is of the form $R^{a} \oplus T^{b} \oplus D$. If $\gamma$ is in $\Gamma_{1}$, then $\gamma$ is constant on cosets of $H_{1}$, as are $\hat{f}$ and $(\phi f)^{\wedge}$, since $f$ and $\phi f$ are supported in $\Gamma_{1}$. Thus elements of $\Gamma_{1}, \hat{f}$, and $(\phi f)^{\wedge}$ naturally define functions on $G_{1}$ : we shall deal largely with these functions.

Choose a neighbourhood $V_{0}$ in $G_{1}$ of the form described in Lemma 1, such that $m\left(V_{0}\right)<1$ and, if $u$ is the function $m\left(V_{0}\right)^{-2} \chi_{V_{0}} * \chi_{V_{0}}$ of Lemma 1 , then

$$
\left|\int_{G_{1}} d x u(x) \gamma(x)(\phi f)^{\wedge}(x)-(\phi f)^{\wedge}(0)\right|<\delta \quad \forall \gamma \in \Lambda_{0} \text {, }
$$

where $\delta$ is a small quantity whose choice will be explained later. This is possible because $(\phi f)^{\wedge}$ is continuous and $\Lambda_{0}$ is compact.

Choose $k$ in $F A_{c}(\Gamma)$ such that

$$
\|(\phi f) * k-\phi f\|_{1}<\delta m\left(V_{0}\right)<\delta
$$

and

$$
\|f * k-f\|_{1}<\delta m\left(V_{0}\right)<\delta
$$

recall that $m\left(V_{0}\right)<1$. Enlarge the support $K_{1}$ of $\hat{k}$ in $G_{1}$ to a compact neighbourhood $K_{2}$ of 0 in $G_{1}$ which contains $V_{0}+V_{0}$. From the theorem on finitely generated abelian groups, $K_{2}$ generates a subgroup of $G_{1}$ of the form $R^{a} \oplus T^{b} \oplus$ $\left(K \oplus Z^{c}\right)$, where $K$ is a finite discrete group and $c$ is a nonnegative integer; $K_{2}$ is therefore contained in a compact subset $K_{3}$ of $G$ of the form $\left[-M_{1}, M_{1}\right]^{a} \oplus$ $T^{b} \oplus K \oplus\left[-M_{1}, M_{1}\right]^{c}$. Let $j$ be a positive integer so large that

$$
((j+1) / j)^{(a+c)}<1+\delta .
$$


Define $M$ to be the positive integer $(j+1) M_{1}$, and let $K_{0}$ be the set $[-M, M]^{a} \oplus$ $T^{b} \oplus K \oplus[-M, M]^{c}$. Let $u$ and $q$ be as in Lemma 1 .

We first prove the inequality (2.4). For $\gamma$ in $\Gamma_{1}$, by the inversion theorem,

$$
\begin{aligned}
\sum_{1}^{\infty} b_{n}(\phi f) * k\left(\gamma+\gamma_{n}\right) & =\int_{K_{0}} d x(\phi f)^{\wedge}(x) \hat{k}(x) \sum_{1}^{\infty} h_{n}\left(\gamma+\gamma_{n}\right)(x) \\
& =\int_{K_{0}} d x(\phi f)^{\wedge}(x) \hat{k}(x) \gamma(x) u(x) .
\end{aligned}
$$

So, for any sufficiently large positive integer $N$ and any $\gamma$ in $\Gamma_{1}$,

$$
\left|\sum_{1}^{N} b_{n}(\phi f) * k\left(\gamma+\gamma_{n}\right)-\int_{G_{1}} d x(\phi f)^{\hat{n}} . \hat{k}(x) \gamma(x) u(x)\right|<\delta .
$$

By (2.7), $\left\|(\phi f)^{\wedge} . \hat{k}-(\phi f)^{\wedge}\right\|_{\infty}<\delta$, whence

$$
\left|\int_{G_{1}} d x\left[(\phi f)^{\wedge}(x) \hat{k}(x)-(\phi f)^{\wedge}(x)\right] \gamma(x) u(x)\right|<\delta .
$$

Thus, from (2.6) and (2.10), for any $\gamma$ in $\Lambda_{0}$,

$$
\left|\sum_{1}^{N} b_{n}(\phi f) * k\left(\gamma+\gamma_{n}\right)-(\phi f)^{\wedge}(0)\right|<3 \delta \text {, }
$$

and therefore

$$
\int_{\Lambda_{0}} d \gamma\left|\sum_{1}^{N} b_{n}(\phi f) * k\left(\gamma+\gamma_{n}\right)-(\phi f)^{\wedge}(0)\right|<3 \delta \mu\left(\Lambda_{0}\right) .
$$

From (2.7), we conclude that

$$
\begin{gathered}
\int_{\Lambda_{0}} d \gamma\left|\sum_{1}^{N} b_{n} \phi\left(\gamma+\gamma_{n}\right) f\left(\gamma+\gamma_{n}\right)-(\phi f)^{\wedge}(0)\right| \\
\leqslant \int_{\Lambda_{0}} d \gamma\left|\sum_{1}^{N} b_{n}[(\phi f) * k-\phi f]\left(\gamma+\gamma_{n}\right)\right|+3 \delta \mu\left(\Lambda_{0}\right) \\
\leqslant \sum_{1}^{N} b_{n} \mid \delta m\left(V_{0}\right)+3 \delta \mu\left(\Lambda_{0}\right) \leqslant \delta+3 \delta \mu\left(\Lambda_{0}\right),
\end{gathered}
$$

and so if $\delta$ is chosen to be less than $\epsilon\left(1+3 \mu\left(\Lambda_{0}\right)\right)^{-1}$, inequality (2.4) is established.

We now estimate $\left\|\Sigma_{1}^{N} b_{n} f\left(\gamma+\gamma_{n}\right)\left(\gamma+\gamma_{n}\right)\right\|_{B_{p}(G)}$. Our first step, the most difficult, is to estimate

$$
\left\|\sum_{1}^{\infty} b_{n}(f * k)\left(\gamma+\gamma_{n}\right) \cdot\left(\gamma+\gamma_{n}\right)\right\|_{B_{p}(G)} .
$$

For any $\gamma$ in $\Gamma_{1}, \Sigma_{1}^{\infty} b_{n}(f * k)\left(\gamma+\gamma_{n}\right) \cdot\left(\gamma+\gamma_{n}\right)$ is constant on cosets of $H_{1}$ in $G$, and so 


$$
\begin{aligned}
\| \sum_{1}^{\infty} b_{n}(f * k)(\gamma & \left.+\gamma_{n}\right) \cdot\left(\gamma+\gamma_{n}\right) \|_{B_{p}(G)} \\
& =\left\|\sum_{1}^{\infty} b_{n}(f * k)\left(\gamma+\gamma_{n}\right) \cdot\left(\gamma+\gamma_{n}\right)\right\|_{B_{p}\left(G_{1}\right)} \\
& =\left\|\sum_{1}^{\infty} b_{n}(f * k)\left(\gamma+\gamma_{n}\right) \cdot \gamma_{n}\right\|_{B_{p}\left(G_{1}\right)} .
\end{aligned}
$$

Denote by $E$ the almost periodic function on $G_{1}$ defined by the formula

$$
E(y)=\sum_{1}^{\infty} b_{n}(f * k)\left(\gamma+\gamma_{n}\right) \gamma_{n}(y) \text {. }
$$

Since $\Sigma_{1}^{\infty}\left|b_{n}\right|<\infty$, we deduce from the inversion theorem that

$$
\begin{aligned}
E(y)=\sum_{1}^{\infty} b_{n} T_{-\gamma}(f * k)\left(\gamma_{n}\right) \cdot \gamma_{n}(y) & =\int_{G_{1}} d x \gamma \hat{f} \hat{k}(x) \sum_{1}^{\infty} b_{n} \gamma_{n}(x+y) \\
& =\int_{G_{1}} d x \gamma \hat{f} \hat{k}(x) q(x+y) .
\end{aligned}
$$

It follows from results of Lohoue [8] that

$$
\left\|\sum_{1}^{\infty} b_{n}(f * k)\left(\gamma+\gamma_{n}\right) \cdot\left(\gamma+\gamma_{n}\right)\right\|_{B_{p}(G)} \leqslant\|\bar{E}\|_{A_{p}\left(\bar{G}_{1}\right)},
$$

$\bar{E}$ being the unique continuous extension of $E$ to $\bar{G}_{1}$. We shall estimate $\|\bar{E}\|_{A_{p}\left(\bar{G}_{1}\right)}$ in terms of $\|\hat{f}\|_{A_{p}(G)}$, and hence obtain the inequality (2.5) from (2.11) and (2.8).

The estimation of $\|\bar{E}\|_{A_{p}\left(\bar{G}_{1}\right)}$ perhaps merits some explanation. It is illuminating to consider the cases $G_{1}=R, T$, and $D$ separately. Suppose first that $G_{1}=T$. Then

$$
E(y)=\int_{G_{1}} d x \gamma \hat{f} \hat{k}(x) q(x+y)=\int_{T} d x \gamma \hat{f} \hat{k}(x) u(x+y) .
$$

From Lemma $1, u$ is in $L^{1}(T)$ and $\|u\|_{1} \leqslant 1$. For any LCA group, $L^{1} * A_{p} \subseteq$ $A_{p}$ and $\|h * k\|_{A_{p}} \leqslant\|h\|_{1}\|k\|_{A_{p}}$. Since also $T=\bar{T}$, it follows that

$$
\|\bar{E}\|_{A_{p}(T)} \leqslant\|\gamma \hat{f} \hat{k}\|_{A_{p}(T)}=\|\hat{f} \hat{k}\|_{A_{p}(T)} \leqslant\|\hat{f}\|_{A_{p}(T)}+\delta,
$$

the last inequality being a consequence of (2.8).

Suppose now that $G_{1}=R$. We can express $\gamma \hat{f} \hat{k}$ as a limit of sums of convolutions of $C_{K_{0}}(R)$-functions. Let us consider one such convolution, $g * h$ say. The function $\bar{q}$ on $\bar{R}$ is a convolution:

$$
\bar{q}=m\left(V_{0}\right)^{-1} \bar{m}(U)^{-1} \chi_{U} \bar{*} \chi_{U}
$$

Informally, $(g * h) *\left(\chi_{U} \bar{*} \chi_{U}\right)=\left(g * \chi_{U}\right) *\left(h * \chi_{U}\right)$ whence 


$$
\left\|(g * h) *\left(\chi_{U} \bar{*} \chi_{U}\right)\right\|_{A_{p}(\bar{R})} \leqslant\left\|f * \chi_{U}\right\|_{L^{p}(\bar{R})}\left\|h * \chi_{U}\right\|_{L^{p^{\prime}}(\bar{R})}
$$

As a function on $R, \chi_{U}$ is a sum of translates of $V_{0}$ :

$$
\chi_{U}=\sum_{x \in I Z} T_{x} \chi_{V_{0}}
$$

Now $g$ say is supported in $[-I / 2, I / 2]$, from which we deduce that

$$
\left\|g * \chi_{U}\right\|_{L} p_{(\bar{R})} \leqslant\|g\|_{L^{p}(R)}\left\|\chi_{U}\right\|_{L^{1}(\bar{R})},
$$

which leads us to the required inequality.

Finally, if $G_{1}=D$, we need, as above, to estimate $\left\|g * \chi_{U}\right\|_{L^{p}(\bar{D})}$ in terms of $\|g\|_{L^{p}(D)}$. As a function on $\bar{D}, g * \chi_{U}$ is a sum of translates of $\chi_{U}$ whose supports are disjoint, i.e.

$$
g * \chi_{U}=\sum g\left(x_{n}\right) T_{x_{n}} \chi_{U}
$$

whence

$$
\left\|g * \chi_{U}\right\|_{L^{p}(\bar{D})}=\left[\sum\left\|g\left(x_{n}\right) T_{x_{n}} \chi_{U}\right\|_{L^{p}(\bar{D})}^{p}\right]^{p^{-1}}=\|g\|_{L^{p}(D)}\left\|\chi_{U}\right\|_{L^{p}(\bar{D})},
$$

which again gives the required estimate.

We now formalise the above ideas. The function $\gamma \hat{f} \hat{k}$ is in $A_{c}\left(G_{1}\right)$, a subspace of $A_{p}\left(G_{1}\right)$, so there exist sequences $\left(g_{n}\right)_{1}^{\infty}$ and $\left(h_{n}\right)_{1}^{\infty}$ of $C_{c}\left(G_{1}\right)$-functions such that

$$
\sum_{1}^{\infty}\left\|g_{n}\right\|_{p}\left\|h_{n}\right\|_{p^{\prime}} \leqslant\|\gamma \hat{f} \hat{k}\|_{A_{p}\left(G_{1}\right)}+\delta
$$

and

$$
\sum_{1}^{\infty} g_{n} * h_{n}=\gamma \hat{f} \hat{k}
$$

Note that, since $\|\gamma\|_{B\left(G_{1}\right)}=\|\gamma\|_{C\left(G_{1}\right)}=1,\|\gamma\|_{B_{p}\left(G_{1}\right)}=1$ for any $\gamma$ in $\Gamma_{1}$. The same equality holds for the inverse of $\gamma$, whence

$$
\|\gamma \hat{f} \hat{k}\|_{A_{p}\left(G_{1}\right)}=\|\hat{f} \hat{k}\|_{A_{p}\left(G_{1}\right)} \leqslant\|\hat{f}\|_{A_{p}\left(G_{1}\right)}+\delta=\|f\|_{A_{p}(G)}+\delta,
$$

the last inequality being a consequence of (2.8). We conclude that

$$
\sum_{1}^{\infty}\left\|g_{n}\right\|_{p}\left\|h_{n}\right\|_{p^{\prime}} \leqslant \cdot\|\hat{f}\|_{A_{p}(G)}+2 \delta .
$$

Recall that $k$ is supported inside a compact subset $K_{2}$ of $G_{1}$ of the form $\left[-M_{1}, M_{1}\right]^{a} \oplus T^{b} \oplus\left(K \oplus\left[-M_{1}, M_{1}\right]^{c}\right)$, and that $K_{0}$ was chosen to be the subset $[-M, M]^{a} \oplus T^{b} \oplus\left(K \oplus[-M, M]^{c}\right)$ of $G_{1}$ where $M=(j+1) M_{1}$. Denote by $\psi_{0}$ and $\psi_{3}$ the characteristic functions of $K_{0}$ and $K_{3}$ respectively, where

$$
K_{3}=\left[M_{1}-M, M-M_{1}\right]^{a} \oplus T^{b} \oplus\left(K \oplus\left[M_{1}-M, M-M_{1}\right]^{c}\right) .
$$

Then $\left\|\psi_{3}\right\|_{1}^{-1} \psi_{0} * \psi_{3}$ takes the value one on $\operatorname{supp}(\hat{k})$, so 


$$
\begin{aligned}
\gamma \hat{f} \hat{k}(x) & =\gamma \cdot \hat{f} \cdot \hat{k} \cdot\left\|\psi_{3}\right\|_{1}^{-1} \psi_{0} * \psi_{3}(x)=\left\|\psi_{3}\right\|_{1}^{-1}\left[\sum_{1}^{\infty} g_{n} * h_{n}\right] \cdot\left[\psi_{0} * \psi_{3}\right](x) \\
& =\left\|\psi_{3}\right\|_{1}^{-1} \sum_{1}^{\infty} \int_{G_{1}} d w\left(T_{w} g_{n} \cdot \psi_{0}\right) *\left(T_{-w} h_{n} \cdot \psi_{3}\right)(x)
\end{aligned}
$$

by a standard argument.

Next, we recall the definition of $E$ :

$$
E(y)=\int_{G_{1}} d x \gamma \cdot \hat{f} \cdot \hat{k}(x) q(x+y) .
$$

We see at once that

$$
E(y)=\int_{G_{1}} d x\left\|\psi_{3}\right\|_{1}^{-1}\left[\sum_{1}^{\infty} \int_{G_{1}} d w\left(T_{w} g_{n} \cdot \psi_{0}\right) *\left(T_{-w} h_{n} \cdot \psi_{3}\right)(x)\right] q(x+y)
$$

$$
=\left\|\psi_{3}\right\|_{1}^{-1} \sum_{1}^{\infty} \int_{G_{1}} d w \int_{G_{1}} d x\left[\left(T_{w} g_{n} \cdot \psi_{0}\right) *\left(T_{-w} h_{n} \cdot \psi_{3}\right)\right](x) q(x+y)
$$

from Fubini's theorem.

From Lemma 1 , for any $g$ and $h$ in $C_{c}\left(G_{1}\right)$, and any $y$ in $G_{1}$,

$$
\begin{array}{rl}
\int_{G_{1}} d x g & * h(x) q(x+y) \\
= & m\left(V_{0}\right)^{-1} \bar{m}(U)^{-1} \int_{G_{1}} d x\left[\int_{G_{1}} d z g(x-z) h(z)\right] \\
& \cdot\left[\int_{\bar{G}_{1}} d \bar{v} \chi_{U}(x+y-\bar{v}) \chi_{U}(\bar{v})\right] \\
=m\left(V_{0}\right)^{-1} \bar{m}(U)^{-1} \int_{\bar{G}_{1}} d \bar{v} G(y-\bar{v}) H(\bar{v}),
\end{array}
$$

where

$$
G(\bar{v})=\int_{G_{1}} d x g(x) \chi_{U}(x+\bar{v}) \quad \forall \bar{v} \in G_{1}
$$

and

$$
H(\bar{v})=\int_{G_{1}} d x h(x) \chi_{U}(x+\bar{v}) \quad \forall \bar{v} \in G_{1}
$$

by Fubini's theorem. Defining the functions $G_{n, w}$ and $H_{n, w}$ by the formulae:

$$
\begin{array}{ll}
G_{n, w}(\bar{v})=\int_{G_{1}} d x\left(T_{w} g_{n} \cdot \psi_{0}\right)(x) \chi_{U}(x+\bar{v}) & \forall \bar{v} \in \bar{G}_{1}, \\
H_{n, w}(\bar{v})=\int_{G_{1}} d x\left(T_{-w} h_{n} \cdot \psi_{3}\right)(x) \chi_{U}(x+\bar{v}) & \forall \bar{v} \in \bar{G}_{1}
\end{array}
$$

it follows from (2.13) that 


$$
E(y)=\left\|\psi_{3}\right\|_{1}^{-1} m\left(V_{0}\right)^{-1} \bar{m}(U)^{-1} \sum_{1}^{\infty} \int_{G_{1}} d w G_{n, w} \bar{*} H_{n, w}(y)
$$

for every $y$ in the dense subgroup $G_{1}$ of $\bar{G}_{1}$. We deduce by a standard argument that

$$
\bar{E}=\left\|\psi_{3}\right\|_{1}^{-1} m\left(V_{0}\right)^{-1} \bar{m}(U)^{-1} \sum_{1}^{\infty} \int_{G_{1}} d w G_{n, w} \bar{*} H_{n, w}
$$

in $A_{p}\left(\bar{G}_{1}\right)$ and that

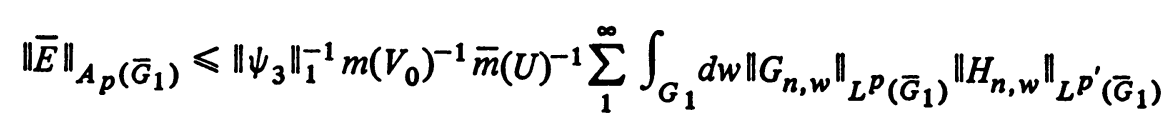

provided, of course, that this last expression is finite. From Hölder's inequality, we conclude that

$$
\|\bar{E}\|_{A_{p}\left(\bar{G}_{1}\right)} \leqslant\left\|\psi_{3}\right\|_{1}^{-1} m\left(V_{0}\right)^{-1} \bar{m}(U)^{-1} \sum_{1}^{\infty}\left[\int_{G_{1}} d w\left\|G_{n, w}\right\|_{L^{p}\left(\bar{G}_{1}\right)}\right]^{p^{-1}}
$$

$$
\cdot\left[\int_{G_{1}} d w\left\|H_{n, w}\right\|_{L^{p^{\prime}}\left(\bar{G}_{1}\right)}^{p^{\prime}}\right]^{p^{\prime-1}}
$$

If $g$ is any bounded measurable function on $G_{1}$ which vanishes off $K_{0}$, and $G$ is the function on $\bar{G}_{1}$ defined by the formula

$$
G(\bar{v})=\int_{G_{1}} d x g(x) \chi_{U}(x+\bar{v}) \quad \forall \bar{v} \in \bar{G}_{1},
$$

then $\|G\|_{1} \leqslant\|g\|_{1}\left\|\chi_{U}\right\|_{1}$ from Fubini's theorem. Moreover,

$$
\begin{aligned}
\|G\|_{\infty} & =\sup \left\{\left|\int_{G_{1}} d x g(x) \chi_{U}(x+\bar{v})\right|: \bar{v} \in G_{1}\right\} \\
& \leqslant \sup \left\{\int_{K_{0}} d x\left\|_{g}\right\|_{\infty} \chi_{U}(x+\bar{v}) \mid: \bar{v} \in G_{1}\right\} \leqslant\|g\|_{\infty} m\left(V_{0}\right)
\end{aligned}
$$

from Lemma 1. By the Riesz-Thorin interpolation theorem, for any $p$ in $[1, \infty]$,

$$
\|G\|_{p} \leqslant\|g\|_{p}\left\|\chi_{U}\right\|_{1}^{p^{-1}} m\left(V_{0}\right)^{p^{\prime}-1}=\|g\|_{p} \bar{m}(U)^{p^{-1}} m\left(V_{0}\right)^{p^{\prime}-1} .
$$

It follows from the inequality (2.14) that

$$
\begin{aligned}
\|\bar{E}\|_{A_{p}\left(\bar{G}_{1}\right)} \leqslant & \left\|\psi_{3}\right\|_{1}^{-1} \sum_{1}^{\infty}\left[\int_{G_{1}} d w\left\|T_{w} g_{n} \cdot \psi_{0}\right\|_{p}^{p}\right]^{p^{-1}} \\
& \cdot\left[\int_{G_{1}} d w\left\|T_{-w} h_{n} \cdot \psi_{3}\right\|_{p^{\prime}}^{p^{\prime}}\right]^{p^{\prime-1}}
\end{aligned}
$$


Now

$$
\begin{aligned}
{\left[\int_{G_{1}} d w\left\|T_{w} g_{n} \cdot \psi_{0}\right\|_{p}^{p}\right]^{p^{-1}} } & =\left[\int_{G_{1}} d w \int_{G_{1}} d x \mid g_{n}(x-w) \psi_{0}(x)^{p}\right]^{p^{-1}} \\
& =\left\|g_{n}\right\|_{p}\left\|\psi_{0}\right\|_{p}
\end{aligned}
$$

by Fubini's theorem, and the translation and reflection invariance of Haar measure. A similar estimate holds for

so

$$
\left[\int_{G_{1}} d w\left\|T_{-w} h_{n} \cdot \psi_{3}\right\|_{p^{\prime}}^{\prime}\right]^{p^{\prime-1}}
$$

$$
\begin{aligned}
& \|\bar{E}\|_{A_{p}\left(\bar{G}_{1}\right)} \leqslant\left\|\psi_{3}\right\|_{1}^{-1}\left\|\psi_{0}\right\|_{p}\left\|\psi_{3}\right\|_{p^{\prime}} \sum_{1}^{\infty}\left\|g_{n}\right\|_{p}\left\|h_{n}\right\|_{p^{\prime}} \\
& \leqslant m\left(K_{0}\right)^{p^{-1}} m\left(K_{3}\right)^{-p^{-1}}\left[\|\hat{f}\|_{A_{p}(G)}+2 \delta\right]
\end{aligned}
$$

from the definitions of $\psi_{0}$ and $\psi_{3}$, and the inequality (2.12). Thus

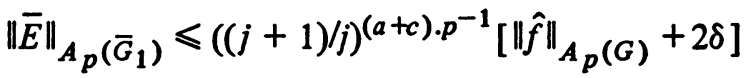

$$
\begin{aligned}
& \leqslant(1+\delta)\left[\|\hat{f}\|_{A_{p}(G)}+2 \delta\right]
\end{aligned}
$$

from the definitions of $K_{0}$ and $K_{3}$, and the inequality (2.9). We conclude, from (2.11), that

$$
\left\|\sum_{1}^{\infty} b_{n}(f * k)\left(\gamma+\gamma_{n}\right) \cdot\left(\gamma+\gamma_{n}\right)\right\|_{B_{p}(G)} \leqslant(1+\delta)\left[\|\hat{f}\|_{A_{p}(G)}+2 \delta\right] .
$$

We can now complete the proof of Lemma 2 very easily. Because

$$
\begin{gathered}
\sum_{1}^{\infty}\left|b_{n}\right|\left|(f * k)\left(\gamma+\gamma_{n}\right)\right| \leqslant \sum_{1}^{\infty}\left|b_{n}\right|\|f * k\|_{\infty}<\infty, \\
\left\|\sum_{1}^{N} b_{n}(f * k)\left(\gamma+\gamma_{n}\right) \cdot\left(\gamma+\gamma_{n}\right)\right\|_{B_{p}(G)} \leqslant(1+\delta)\left[\|\hat{f}\|_{A_{p}(G)}+3 \delta\right]
\end{gathered}
$$

for all sufficiently large $N$. Consequently,

$$
\begin{aligned}
& \left\|\sum_{1}^{N} b_{n} f\left(\gamma+\gamma_{n}\right) \cdot\left(\gamma+\gamma_{n}\right)\right\|_{B_{p}(G)} \\
& \quad \leqslant\left\|\sum_{1}^{N} b_{n}(f-f * k)\left(\gamma+\gamma_{n}\right) \cdot\left(\gamma+\gamma_{n}\right)\right\|_{B_{p}(G)}+(1+\delta)\left[\|\hat{f}\|_{A_{p}(G)}+3 \delta\right] \\
& \quad \leqslant \sum_{1}^{N}\left|b_{n}\right|\left|(f-f * k)\left(\gamma+\gamma_{n}\right)\right|+(1+\delta)\left[\|\hat{f}\|_{A_{p}(G)}+3 \delta\right],
\end{aligned}
$$


and so

$$
\begin{aligned}
& \int_{\Lambda_{0}} d \gamma\left\|\sum_{1}^{N} b_{n} f\left(\gamma+\gamma_{n}\right) \cdot\left(\gamma+\gamma_{n}\right)\right\|_{B_{p}(G)} \\
& \quad \leqslant \sum_{1}^{N}\left|b_{n}\right|\|f-f * k\|_{1}+(1+\delta) \mu\left(\Lambda_{0}\right)\left[\|\hat{f}\|_{A_{p}(G)}+3 \delta\right] \\
& \quad \leqslant \delta+(1+\delta) \mu\left(\Lambda_{0}\right)\left[\|\hat{f}\|_{A_{p}(G)}+3 \delta\right]
\end{aligned}
$$

by (2.8). Therefore, if $\delta$ be chosen to satisfy the conditions

$$
\delta\left[1+3 \mu\left(\Lambda_{0}\right)\right]<\epsilon
$$

and

$$
\delta+(1+\delta) \mu\left(\Lambda_{0}\right)\left[\|\hat{f}\|_{A_{p}(G)}+3 \delta\right]<\mu\left(\Lambda_{0}\right)\|\hat{f}\|_{A_{p}(G)}+\epsilon,
$$

and $N$ is then chosen so that both (2.10) and (2.15) are satisfied, the lemma is proved.

Proof of Theorem 2 (continued). To prove Theorem 2, we show that the inequality (2.1) is satisfied.

Suppose that $f$ is a bounded measurable function vanishing outside the compact subset $\Lambda_{0}$ of $\Lambda$, and that $\epsilon$ is any small positive quantity. By Lemma 2, there exists a trigonometric polynomial $\Sigma_{1}^{N} b_{n} \gamma_{n}$ such that

$$
\int_{\Lambda_{0}} d \gamma\left|\sum_{1}^{N} b_{n} \phi\left(\gamma+\gamma_{n}\right) f\left(\gamma+\gamma_{n}\right)-(\phi f)^{\wedge}(0)\right|<\epsilon^{2} \mu\left(\Lambda_{0}\right)
$$

and

$$
\int_{\Lambda_{0}} d \gamma\left\|\sum_{1}^{N} b_{n} \cdot f\left(\gamma+\gamma_{n}\right) \cdot\left(\gamma+\gamma_{n}\right)\right\|_{B_{p}(G)}<\mu\left(\Lambda_{0}\right)\left[\|\hat{f}\|_{A_{p}(G)}+\epsilon\right]
$$

Define the measurable subset $\Lambda_{1}$ of $\Lambda_{0}$ by the formula

$$
\Lambda_{1}=\left\{\gamma \in \Lambda_{0}:\left|\sum_{1}^{N} b_{n} \cdot \phi\left(\gamma+\gamma_{n}\right) f\left(\gamma+\gamma_{n}\right)-(\phi f)^{\wedge}(0)\right|>\epsilon\right\}
$$

Now

$$
\epsilon \mu\left(\Lambda_{1}\right) \leqslant \int_{\Lambda_{1}} d \gamma\left|\sum_{1}^{N} b_{n} \cdot \phi\left(\gamma+\gamma_{n}\right) f\left(\gamma+\gamma_{n}\right)-(\phi f)^{\wedge}(0)\right|
$$

so $\epsilon \mu\left(\Lambda_{1}\right)<\epsilon^{2} \mu\left(\Lambda_{0}\right)$ from (2.16). We deduce that $\mu\left(\Lambda_{1}\right)<\epsilon \mu\left(\Lambda_{0}\right)$.

Similarly, defining $\Lambda_{2}$ by the formula 


$$
\Lambda_{2}=\left\{\gamma \in \Lambda_{0}:\left\|\sum_{1}^{N} b_{n} \cdot f\left(\gamma+\gamma_{n}\right)\left(\gamma+\gamma_{n}\right)\right\|_{B_{p}(G)}>(1-\epsilon)^{-1}\left[\|\hat{f}\|_{A_{p}(G)}+\epsilon\right]\right\}
$$

we can deduce that $\mu\left(\Lambda_{2}\right)<(1-\epsilon) \mu\left(\Lambda_{0}\right)$. Consequently, $\mu\left(\Lambda_{1} \cup \Lambda_{2}\right)<\mu\left(\Lambda_{0}\right)$; take $\gamma_{0}$ in $\Lambda_{0} \backslash\left(\Lambda_{1} \cup \Lambda_{2}\right)$. Then

$$
\left|\sum_{1}^{N} b_{n} \phi\left(\gamma_{0}+\gamma_{n}\right) f\left(\gamma_{0}+\gamma_{n}\right)-(\phi f)^{\wedge}(0)\right| \leqslant \epsilon
$$

and

$$
\left\|\sum_{1}^{N} b_{n} f\left(\gamma_{0}+\gamma_{n}\right) \cdot\left(\gamma_{0}+\gamma_{n}\right)\right\|_{B_{p}(G)} \leqslant(1-\epsilon)^{-1}\left[\|\hat{f}\|_{A_{p}(G)}+\epsilon\right] .
$$

Let $\Lambda_{3}$ be the set $\Lambda_{0} \cap\left\{\gamma_{0}+\gamma_{n}: n=1,2, \ldots, N\right\}$. The hypothesis of the theorem implies that there is a trigonometric polynomial $t$ on $\Gamma$ such that $\left\|_{t}\right\|_{M_{p}} \leqslant C$ and

$$
|t(\gamma)-\phi(\gamma)|<\epsilon\left[\sum_{1}^{N}\left|b_{n}\right| \cdot\|f\|_{\infty}\right]^{-1} \quad \forall \gamma \in \Lambda_{3} .
$$

Since $f\left(\gamma_{0}+\gamma_{n}\right)$ vanishes unless $\gamma_{0}+\gamma_{n}$ is in $\Lambda_{0}$,

$$
\left|\sum_{1}^{N} b_{n} t\left(\gamma_{0}+\gamma_{n}\right) f\left(\gamma_{0}+\gamma_{n}\right)-(\phi f) \cdot(0)\right| \leqslant 2 \epsilon
$$

from (2.18). Therefore

$$
\begin{aligned}
\left|\int_{\Lambda} d \gamma \phi(\gamma) f(\gamma)\right| & \leqslant\left|\sum_{1}^{N} b_{n} t\left(\gamma_{0}+\gamma_{n}\right) f\left(\gamma_{0}+\gamma_{n}\right)\right|+2 \epsilon \\
& \leqslant\left|\int_{G} d \hat{t}(x) \sum_{1}^{N} b_{n} f\left(\gamma_{0}+\gamma_{n}\right)\left(\gamma_{0}+\gamma_{n}\right)(x)\right|+2 \epsilon .
\end{aligned}
$$

However $\hat{t}$ is a discrete measure with finite support, and $A_{p}(G)$ contains a local unit, so

$$
\left|\int_{\Lambda} d \gamma \phi(\gamma) f(\gamma)\right| \leqslant\|t\|_{M_{p}}\left\|\sum_{1}^{N} b_{n} f\left(\gamma_{0}+\gamma_{n}\right)\left(\gamma_{0}+\gamma_{n}\right)\right\|_{B_{p}(G)}+2 \epsilon .
$$

Taking (2.19) into account, we deduce that

$$
\left|\int_{\Lambda} d \gamma \phi(\gamma) f(\gamma)\right| \leqslant C(\dot{1}-\epsilon)^{-1}\left[\|\hat{f}\|_{A_{p}(G)}+\epsilon\right]+2 \epsilon,
$$

but $\epsilon$ can be arbitrarily small, proving the inequality (2.1) and thereby the theorem.

The author wishes to thank Professor G. I. Gaudry of the Flinders Universi- 
ty of South Australia, under whose auspices this paper was prepared as part of work for a Ph.D. degree, for his aid.

\section{REFERENCES}

1. M. G. Cowling, Spaces $A_{p}^{q}$ and $L^{p}-L^{q}$ Fourier multipliers, Doctoral Dissertation, Flinders University of South Australia, Bedford Park, 1974.

2. K. de Leeuw and C. Herz, An invariance property of spectral synthesis, Illinois J. Math. 9 (1965), 220-229. MR 31 \#3787.

3. R. Doss, Approximations and representations for Fourier transforms, Trans. Amer. Math. Soc. 153 (1971), 211-221. MR 42 \#3494.

4. N. Dunford and J. T. Schwartz, Linear operators. I: General theory, Pure and Appl. Math., vol. 7, Interscience, New York, 1958. MR 22 \#8302.

5. A. Figà-Talamanca, Translation invariant operators in $L^{p}$, Duke Math. J. 32 (1965), 495-501. MR 31 \#6095.

6. C. Herz, Remarques sur la note précédente de M. Varopoulos, C. R. Acad. Sci. Paris 260 (1965), 6001-6004. MR 31 \#6096.

7. C. Herz, Séminaire Orsay harmonique, juin 1970.

8. N. Lohoué, La synthèse des convoluteurs sur un group abélien localement compact, C. R. Acad. Sci. Paris Ser. A-B 272 (1971), A27-A29. MR 43 \#820.

9. S. Saeki, Translation invariant operators on groups, Tôhoku Math. J. (2) 22 (1970), 409-419. MR 43 \#815.

DEPARTMENT OF MATHEMATICS, UNIVERSITY OF BRITISH COLUMBIA, VANCOUVER 8, BRITISH COLUMBIA, CANADA 